\title{
Aspectos sociocognitivos como eventos estressantes na saúde mental em grupos étnicos e minoritários no Brasil
}

\author{
Aspects of social cognition as stressing events in the mental health of \\ ethnic and minority groups in Brazil
}

\author{
Diana Ramos-Oliveira* \\ Pilar Magnavita \\ Felipe Santos de Oliveira \\ Universidade Católica de Petrópolis, Río de Janeiro, Brasil \\ (Rec.: octubre de 2016 - Acept.: abril de 2017)
}

\begin{abstract}
Resumo
Esta revisão sistemática teve como objetivo avaliar as evidências na literatura dos aspectos sociocognitivos como eventos estressantes para a saúde mental em grupos étnicos e minoritários. As bases de dados pesquisadas foram PubMed, SCIELO e Pepsic. Através dos descritores, a aplicação dos critérios de seleção dos estudos, e depois de ler os títulos e resumos, chegamos a um resultado de 792 estudos; e depois de ler os textos 532 artigos foram excluídos por duplicata, totalizando 32 artigos a serem analisados. Os resultados mostraram que a natureza e persistência dos aspectos sociocognitivos, como racismo, a discriminação e o preconceito e as múltiplas maneiras em que estes podem impactar negativamente a saúde de grupos étnico-raciais, desde as políticas e práticas de acesso aos recursos, estendendo-se aos estereótipos negativos, sustentados pelo racismo cultural que fomentam respostas psicológicas prejudiciais à saúde. Ademais os estudos revelaram que a experiência subjetiva de discriminação racial é um tipo de experiência de vida estressante, mas historicamente negligenciado. Conclui-se que em contextos altamente desiguais e adversos, como é o caso do Brasil, e com evidências de preconceito, racismo e discriminação no âmbito da saúde, as pessoas padecem de desconforto psicológico por suas pertenças étnico-raciais e por ser parte de grupos minoritários.
\end{abstract}

Palavras-chave: cognição social, saúde mental, etnicidade.

\section{Abstract}

This systematic analysis aims to evaluate the evidence in literature regarding socio-cognitive aspects as stressful events for mental health in ethnic and minority groups. The databases tackled were PubMed, SCIELO and Pepsic. After reaching a total of 792 studies through descriptors, selection criteria, and reading different titles and abstracts, 532 duplicated articles were excluded and finally 32 articles were analyzed. The results show that some sociocognitive aspects such as racism, discrimination and prejudice affect the health of ethnic and racial groups in multiple negative ways. When the policies and practices that control access to resources are based on negative stereotypes promoted by cultural racism, this results in in harmful psychological responses to health. Historically, studies have shown that even though racial discrimination is seen as a stressful, subjective situation, it has been neglected so far. In highly inequitable and adverse environments, such as Brazil, people suffer from a psychological distress due to their ethnic or racial affiliation, or for being part of a minority group as a consequence of the same discrimination and existent prejudice they are victims of.

Keywords: social cognition, mental health, ethnicity.

\footnotetext{
* Correspondencia a: Diana Ramos-Oliveira. Universidade Católica de Petrópolis, Rio de Janeiro, Brasil. Endereço: Campus Benjamin Constant - BC, Rua Benjamin Constant,213, Centro, Petrópolis-RJ. CEP: 25610-130. E-mail: diana.oliveira@ucp.br.
} 


\section{Resumen}

La presente revisión tuvo como objetivo evaluar la literatura basada en las evidencias correspondientes a los aspectos socio-cognitivos como eventos estresantes para la salud mental en los grupos étnicos y minoritarios. La búsqueda tuvo lugar en las bases de datos PubMed, SCIELO y Pepsic. Para ello, se utilizaron los descriptores y la aplicación de criterios de selección. Tras la lectura de títulos y resúmenes, se llegó a la selección preliminar de 792 estudios, siendo excluidos 532 artículos repetidos. Finalmente, quedaron un total de 32 artículos a analizar. Los resultados mostraron que aquellos aspectos socio-cognitivos tales como el racismo, la discriminación y los prejuicios afectan negativamente y de múltiples formas a la salud de los grupos étnicos-raciales. Cuando las políticas y prácticas que controlan el acceso a los recursos se encuentran basadas en estereotipos negativos promovidos por el racismo cultural, el resultado es la generación de respuestas psicológicas nocivas para la salud. Los estudios han demostrado que, históricamente, a pesar de que la discriminación racial es una experiencia subjetiva estresante, no ha sido investigada suficientemente. En entornos altamente desiguales y adversos, tales como Brasil, las personas sufren malestar psicológico debido a su filiación étnico-racial o por el hecho de pertenecer a grupos minoritarios como consecuencia de la discriminación y los prejuicios existentes.

Palabras clave: cognición social, salud mental, etnicidad.

\section{Introdução}

Depressão e ansiedade são distúrbios comuns de saúde mental, no entanto, os riscos de depressão e ansiedade não são distribuídos igualmente (Watson, Roberts \& Saunders, 2012). As diferenças raciais/étnicas na prevalência de sintomas de depressão e ansiedade tèm sido documentada em diversos estudos utilizando amostras de adultos e alguns com adolescentes (Blazer, Kessler, McGonagle \& Swartz, 1994; Brown, Eaton \& Sussman, 1990; Kessler et al., 1994; McLaughlin, Hilt \& Nolen-Hoeksema, 2007).

Esses tipos de estudos têm importantes implicações nos esforços de prevenção e intervenção, mas ainda assim, estudos como estes têm ressaltado os desafios de se fazer um diagnóstico psiquiátrico no contexto da raça e etnia (Watkins, Assari \& JohnsonLawrence, 2015). Além disso, desigualdades também no acesso aos cuidados de saúde mental são proeminentes e afetam desproporcionalmente os membros grupos raciais/étnicos e minoritários. Compreender os mecanismos que levam a essas desigualdades ou as disparidades também é fundamental para informar as políticas de saúde mental e melhorar as diretrizes práticas para 0 tratamento de doenças mentais nessas populações.

No caso da depressão, segundo um estudo do National Center for Health Statistics de 1988 a 1994 nos Estados Unidos (Riolo, Nguyen, Greden \& King, 2005), a prevalência de transtorno depressivo maior foi significativamente elevada em pessoas brancas do que em afro-americanos e mexicano-americanos; o padrão oposto foi encontrado para transtorno distímico. Entre grupos étnicos/raciais, a pobreza foi um fator de risco significativo para o transtorno depressivo maior, mas interações significativas ocorreram entre raça/etnia, gênero e educação em relação à prevalência de transtorno distímico. Este mesmo estudo adverte que os dados da literatura cientifica sobre a prevalência de depressão por raça/etnia são contraditórios, sendo que alguns estudos relatam taxas mais elevadas de transtorno depressivo maior em afro-americanos se comparados a indivíduos brancos, enquanto outros apresentam taxas mais baixas ou equivalentes nestes indivíduos.
Em relação a transtornos ansiosos, dados do Collaborative Psychiatric Epidemiology Studies (CPES) (Asnaani, Richey, Dimaite, Hinton \& Hofmann, 2010) apontam que as pessoas brancas eram mais propensas a ser diagnosticados com transtorno de ansiedade social, transtorno de ansiedade generalizada e transtorno do pânico do que afro-americanos, hispânicos e asiáticos. Os afro-americanos com mais frequência preencheram os critérios para transtorno de estresse póstraumático do que os brancos, hispânicos e asiáticos. Asiáticos também foram menos susceptíveis de satisfazer os diagnósticos para transtorno de ansiedade generalizada e transtorno de estresse pós-traumático do que os hispanoamericanos que por sua vez, eram menos propensos a receber diagnósticos de transtorno de ansiedade social, transtorno de ansiedade generalizada, transtorno do pânico, e estresse póstraumático do que brancos. Os resultados sugerem uma necessidade que a raça e a etnia devam ser consideradas na atribuição de um diagnóstico de transtorno de ansiedade.

Nas diferenças étnicas dos transtornos mentais, tanto sintomas internalizantes quantos externalizantes (Achenbach, 1991 como ele citou em Nakamura, Ebesutani, Bernstein \& Chorpita, 2009) estão associados à discriminação racial e étnica, experiência comum mesmo em países desenvolvidos como os Estados Unidos (Juang, Ittel, Hoferichter \& Gallarin, 2016). Embora nem todos sofram as mesmas consequências negativas ou com a mesma intensidade, o modo como os indivíduos gerenciam suas respostas emocionais as experiências de discriminação podem ser resultado de alguma variação individual sobre os efeitos psicológicos e na saúde de uma maneira geral (Juang et al., 2016). Mas a concentração apenas em aspectos individuais não são suficientes para resolver a nocividade da discriminação e não necessariamente podem oferecer um melhor entendimento e ponto de acesso para a intervenção.

As pesquisas epidemiológicas vêm apresentando evidências sobre processo de discriminação como um fator determinante na saúde da população, iniciada desde a sua infância. Esta questão aponta para duas consequências - discriminação 
econômica e o incremento de insultos acumulados na vida cotidiana, e às vezes as experiências violentas de ser tratado como um cidadão de segunda classe, em cada e todos os níveis da economia (Krieger, 1999). Alguns estudos têm demonstrado que determinados aspectos sociocognitivos atuam como preditores de eventos estressantes, por exemplo, o preconceito e os estereótipos em relação a determinados grupos étnicos, levando a que os indivíduos busquem uma maior identificação étnica para minimizar o malestar mental e a depressão (Ramos-Oliveira, 2009; Ramos-Oliveira, Pankalla \& Cabecinhas, 2012). Esta relação de aspectos sociocognitivos como fatores estressantes na vida dos indivíduos se vê refletido nos estudos de Kelaher, Paul, Lambert, Ahmad e Smith (2008) demonstrando como a exposição à discriminação se associa com níveis mais altos de ansiedade, de preocupação ou depressão. Estes resultados apoiam a afirmação de que a discriminação racial pode desempenhar um papel importante na saúde mental, ademais, pode ter efeitos indiretos na saúde.

As desigualdades raciais/étnicas na saúde mental apresentam ser bem documentadas na literatura estrangeira, entretanto, sabe-se muito pouco sobre estes eventos psicosociocognitivos estressantes e seus efeitos na saúde mental no Brasil. Este artigo propõe uma revisão da literatura, para entender se e como as disparidades emergem e como as pessoas implicadas se veem afetadas na sua saúde mental. Neste contexto, o presente estudo tem por objetivo a) apresentar uma revisão sistemática sobre como a discriminação, o preconceito e os estereótipos estão relacionados com a saúde mental em certos grupos; b) conhecer os estudos na área da Psicologia sobre a associação entre estes aspectos sociocognitivos como eventos estressantes na saúde mental, ou seja, das diferenças étnicas e a influência da presença de racismo no curso clínico dos transtornos depressivos e ansiosos experimentados pelos brasileiros.

\section{Método}

\section{Busca da Literatura e Critério de inclusão}

Realizou-se um estudo exploratório de revisão da literatura, por pesquisas que relacionavam os aspectos psicossociais e de saúde coletiva, como os estereótipos negativos, preconceito e discriminação em grupos étnicos e minoritários e a saúde mental no Brasil. Foram considerados artigos publicados no período entre 2006 e 2016, a pesquisa foi realizada nas seguintes bases de dados: PubMed, Scielo (Scientific Electronic Library Online) e Pepsic.

Correspondentes ao tema proposto foram encontrados 792 artigos científicos. Quinhentos e trinta e dois foram descartados por repetição, sendo o total de objeto desta análise 32 artigos científicos (Tabela 1), sendo excluídas monografias, dissertações e teses.

Inicialmente realizou-se uma consulta aos DeCS Descritores em Ciências da Saúde da Biblioteca
Virtual em Saúde do Lilacs, para uso adequado dos termos nas buscas dos artigos. Os descritores foram combinados para melhor definir as buscas, resultando em 11 combinações com os termos: "estereótipos","racismo", "saúde mental" "depressão", "ansiedade" e "Brasil", estas combinações tanto no idioma Português quanto no Inglês, usando a conjunção AND.

\section{Resultados e discussão}

\section{seleção dos estudos}

Os estudos foram encontrados da seguinte maneira: 601 estudos na base de dados Pubmed; 191 na Scielo e nenhum na Pepsic.

Depois de ler os títulos e os resumos encontrados, foram selecionados estudos com as seguintes características: grupos étnicos, grupos em vulnerabilidade social, grupos minoritários, estudos no campo da psicologia, saúde pública ou coletiva ficando assim incluídos no estudo. Todos os artigos selecionados foram em amostra com indivíduos que eram saudáveis e indivíduos que participavam de alguma intervenção clínica por desconforto na saúde mental, universitários, casais chefe de família.

\section{Resultados avaliados}

A triagem dos artigos levou em consideração a presença de eventos relacionados com a Cognição Social como eventos estressantes para a saúde mental, tal como, discriminação por pertença a grupos étnicos e minoritários e as repercussões na saúde mental; vulnerabilidade social, preconceito, a percepção social e as condições socioeconômicas.

\section{Características dos estudos}

A pesquisa que tratou de avaliar como os aspectos sociocognitivos atuam como eventos estressantes em indivíduos de grupos étnicos ou minoritários. As populações da amostra variavam entre 12 até 6.349 .

Periódicos especializados em saúde pública, epidemiologia e psicologia que tinham publicado sobre a temática, no caso da psicologia na sua grande maioria os artigos eram de revisão sistemática. Os periódicos Brasileiros apresentavam boa qualidade com estudos randomizados e, com ensaios clínicos.

Majoritariamente as pesquisas empíricas que buscaram a relação saúde mental como evento estressante por conta da raça/etnia nos processos sociocognitivos, grupos em situação de vulnerabilidade social, seu acesso aos recursos e o comprometimento da saúde mental pelas situações traumáticas e adversas vivenciadas pelos indivíduos.

A metodologia na sua maioria situou-se entre revisões sistemáticas, estudos cross-sectional e de coorte que são os mais utilizados na área de saúde. 


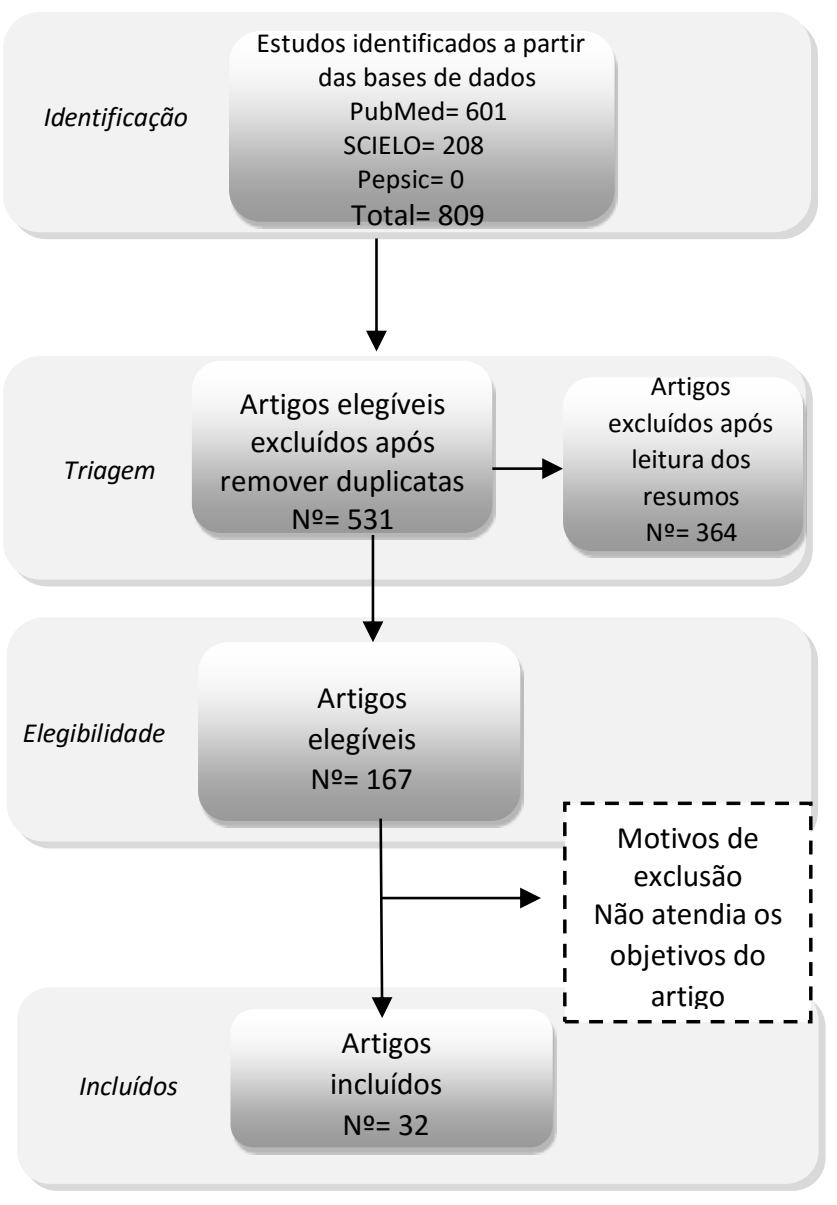

Figura 1. Fluxograma das diferentes fases da Revisão sistemática

Tabela 1. Pesquisas que apontam a relação de aspectos sociocognitivos como eventos estressantes para a saúde mental de grupos étnicos ou minoritários no Brasil

\begin{tabular}{|c|c|c|c|c|c|c|}
\hline & Ano & Periódico & Autor & Título & Objetivos & Principais Resultados \\
\hline 1 & 2006 & $\begin{array}{l}\text { Revista de } \\
\text { Psicologia } \\
\text { Política }\end{array}$ & Vilhena & $\begin{array}{l}\text { A violência da } \\
\text { cor: Sobre } \\
\text { racismo, } \\
\text { alteridade e } \\
\text { intolerância }\end{array}$ & $\begin{array}{l}\text { Revisar e Discutir } \\
\text { algumas das } \\
\text { consequências } \\
\text { psíquicas da } \\
\text { intolerância racial. }\end{array}$ & $\begin{array}{l}\text { Aponta para o poder de } \\
\text { persuasão que fixa negros, } \\
\text { trabalhadores pobres, } \\
\text { desempregados, indigentes, } \\
\text { loucos, mulheres, etc. em } \\
\text { identidades coletivas e } \\
\text { serializadas. }\end{array}$ \\
\hline
\end{tabular}

\begin{tabular}{|c|c|c|c|c|c|c|}
\hline 2 & 2007 & $\begin{array}{l}\text { Rev } \\
\text { Saúde } \\
\text { Pública }\end{array}$ & $\begin{array}{l}\text { Ferriolli, } \\
\text { Marturano \& } \\
\text { Puntel }\end{array}$ & $\begin{array}{l}\text { Contexto } \\
\text { familiar e } \\
\text { problemas de } \\
\text { saúde mental } \\
\text { infantil no } \\
\text { Programa } \\
\text { Saúde da } \\
\text { Família }\end{array}$ & $\begin{array}{l}\text { Analisar a associação } \\
\text { entre variáveis do } \\
\text { contexto familiar e o } \\
\text { risco de problemas } \\
\text { emocionais/comportam } \\
\text { entais em crianças } \\
\text { cadastradas em } \\
\text { Programa Saúde da } \\
\text { Família }\end{array}$ & $\begin{array}{l}\text { O estresse materno foi } \\
\text { também um fator de risco } \\
\text { para sintomas de } \\
\text { ansiedade/depressão. }\end{array}$ \\
\hline 3 & 2007 & $\begin{array}{l}\text { J Bras } \\
\text { Psiquiatr }\end{array}$ & $\begin{array}{l}\text { Miyasaka et } \\
\text { al. }\end{array}$ & $\begin{array}{l}\text { Migration and } \\
\text { mental health: } \\
\text { Japanese }\end{array}$ & $\begin{array}{l}\text { Comparar o perfil } \\
\text { sociodemográfico e } \\
\text { diagnósticos dos }\end{array}$ & $\begin{array}{l}\text { O grupo que retornou ao } \\
\text { Brasil era principalmente de } \\
\text { homens, não casados, que }\end{array}$ \\
\hline
\end{tabular}




\begin{tabular}{|c|c|c|c|c|c|c|}
\hline & Ano & Periódico & Autor & Título & Objetivos & Principais Resultados \\
\hline 7 & $\Gamma$ & & $\Gamma$ & $\begin{array}{l}\text { Brazilians in } \\
\text { Japan and in } \\
\text { Brazil }\end{array}$ & $\begin{array}{l}\text { pacientes ambulatoriais } \\
\text { brasileiros } \\
\text { descendentes de } \\
\text { japoneses que } \\
\text { permaneceram no } \\
\text { Japão com os que } \\
\text { retornaram ao Brasil }\end{array}$ & $\begin{array}{l}\text { viviam sós no Japão, tiveram } \\
\text { uma breve estada neste país } \\
\text { e foram classificados no } \\
\text { grupo de esquizofrenia. O } \\
\text { grupo no Japão era } \\
\text { principalmente constituído } \\
\text { de mulheres, casadas, } \\
\text { morando com familiares ou } \\
\text { amigos, estada longa no } \\
\text { Japão e foram classificadas } \\
\text { no grupo de ansiedade. } \\
\text { Morar em família e ter uma } \\
\text { rede de amigos é muito } \\
\text { importante para a saúde } \\
\text { mental no contexto avaliado }\end{array}$ \\
\hline 4 & 2008 & $\begin{array}{l}\text { Psychiatri } \\
\text { c Reseach }\end{array}$ & $\begin{array}{l}\text { Berlim, } \\
\text { McGirr \& } \\
\text { Fleck }\end{array}$ & $\begin{array}{l}\text { Can } \\
\text { sociodemogra } \\
\text { phic and } \\
\text { clinical } \\
\text { variables } \\
\text { predict the } \\
\text { quality of life of } \\
\text { outpatients } \\
\text { with major } \\
\text { depression? }\end{array}$ & $\begin{array}{l}\text { Conhecer a relação } \\
\text { entre qualidade de vida } \\
\text { e um número de fatores } \\
\text { potencialmente } \\
\text { mediadores, tais como } \\
\text { variáveis } \\
\text { sociodemográficas e } \\
\text { clínicas. }\end{array}$ & $\begin{array}{l}\text { A idade, etnicidade, } \\
\text { comorbidades psiquiátricas, } \\
\text { sintomas psicóticos, e } \\
\text { suicídio foram preditores } \\
\text { ligeiramente significativos } \\
\text { para a qualidade de vida. }\end{array}$ \\
\hline 5 & 2009 & $\begin{array}{l}\text { Cad. } \\
\text { Saúde } \\
\text { Pública }\end{array}$ & $\begin{array}{c}\text { Kang, } \\
\text { Razzouk, } \\
\text { Mari \& } \\
\text { Shirakawa }\end{array}$ & $\begin{array}{l}\text { The mental } \\
\text { health of } \\
\text { Korean } \\
\text { immigrants in } \\
\text { São Paulo, } \\
\text { Brazil }\end{array}$ & $\begin{array}{l}\text { Verificar a frequência } \\
\text { de transtornos } \\
\text { psiquiátricos em } \\
\text { comunidade de uma } \\
\text { imigrantes coreanos na } \\
\text { cidade de São Paulo }\end{array}$ & $\begin{array}{l}\text { Os inmigrantes coreanos } \\
\text { apresentam mais transtornos } \\
\text { psiquiátricos do que a } \\
\text { população coreana na } \\
\text { Coréia, especialmente } \\
\text { transtorno de estresse pós- } \\
\text { traumático. }\end{array}$ \\
\hline 6 & 2010 & $\begin{array}{c}\text { Sau. \& } \\
\text { Transf. } \\
\text { Soc }\end{array}$ & $\begin{array}{l}\text { Bastos, } \\
\text { Celeste, } \\
\text { Faerstein \& } \\
\text { Barros }\end{array}$ & $\begin{array}{l}\text { Discriminação } \\
\text { racial e saúde: } \\
\text { uma revisão } \\
\text { sistemática de } \\
\text { escalas com } \\
\text { foco em suas } \\
\text { propriedades } \\
\text { psicométricas }\end{array}$ & $\begin{array}{l}\text { Revisar } \\
\text { sistematicamente a } \\
\text { literatura sobre escalas } \\
\text { de discriminação racial, } \\
\text { com vistas a descrever } \\
\text { seus processos de } \\
\text { desenvolvimento }\end{array}$ & $\begin{array}{l}\text { A necessidade de considerar } \\
\text { outras formas de tratamento } \\
\text { discriminatório como } \\
\text { exposições danosas à saúde } \\
\text { igualmente importantes e a } \\
\text { ideia de um instrumento } \\
\text { universal, adaptável a } \\
\text { diferentes contextos } \\
\text { socioculturais. }\end{array}$ \\
\hline 7 & 2011 & $\begin{array}{l}\text { Estudos } \\
\text { de } \\
\text { Psicologia }\end{array}$ & $\begin{array}{l}\text { Faro \& } \\
\text { Pereira }\end{array}$ & $\begin{array}{l}\text { Raça, } \\
\text { Racismo e } \\
\text { Saúde: A } \\
\text { desigualdade } \\
\text { Social da } \\
\text { Distribuição do } \\
\text { Estresse }\end{array}$ & $\begin{array}{l}\text { Revisar a literatura e } \\
\text { levantar evidências da } \\
\text { relação entre racismo e } \\
\text { saúde a partir dos } \\
\text { estudos sobre o } \\
\text { estresse }\end{array}$ & $\begin{array}{l}\text { Indícios que há a distribuição } \\
\text { social desigual das } \\
\text { experiências estressoras } \\
\text { conforme a raça, } \\
\text { principalmente quando elas } \\
\text { se associam a situações de } \\
\text { racismo, cujos efeitos } \\
\text { deletérios parecem } \\
\text { configurá-las como } \\
\text { determinantes psicossociais } \\
\text { da saúde. }\end{array}$ \\
\hline 8 & 2011 & $\begin{array}{l}\text { Ciência \& } \\
\text { Saúde } \\
\text { Coletiva }\end{array}$ & $\begin{array}{l}\text { de Andrade, } \\
\text { Assumpção, } \\
\text { Abdalla \& } \\
\text { da Silva }\end{array}$ & $\begin{array}{l}\text { Prevalência de } \\
\text { transtornos } \\
\text { psiquiátricos } \\
\text { em jovens } \\
\text { infratores na } \\
\text { cidade do Rio } \\
\text { de Janeiro }\end{array}$ & $\begin{array}{l}\text { Avaliar a prevalência de } \\
\text { transtornos mentais em } \\
\text { adolescentes sob } \\
\text { medida socioeducativa, } \\
\text { considerando como } \\
\text { hipótese sua diferença } \\
\text { entre gêneros com }\end{array}$ & $\begin{array}{l}\text { Os dados sugerem que se } \\
\text { as autoridades em saúde } \\
\text { pública que tanto a detecção } \\
\text { quanto o tratamento precoce } \\
\text { de transtornos psiquiátricos } \\
\text { na infância podem ajudar na }\end{array}$ \\
\hline
\end{tabular}




\begin{tabular}{|c|c|c|c|c|c|c|}
\hline & Ano & Periódico & Autor & Título & Objetivos & Principais Resultados \\
\hline & & & & $\begin{array}{l}\text { (RJ, Brasil): } \\
\text { estudo de } \\
\text { gênero e } \\
\text { relação com a } \\
\text { gravidade do } \\
\text { delito }\end{array}$ & $\begin{array}{l}\text { base no tipo de delito } \\
\text { cometido. }\end{array}$ & $\begin{array}{l}\text { preven- ção de atos } \\
\text { infratores }\end{array}$ \\
\hline 9 & 2011 & $\begin{array}{c}\text { Soc } \\
\text { Psychiatry } \\
\text { Psychiatr } \\
\text { Epidemiol }\end{array}$ & $\begin{array}{l}\text { Curto, } \\
\text { Paula, do } \\
\text { Nascimen- } \\
\text { to, Murray \& } \\
\text { Bordin }\end{array}$ & $\begin{array}{l}\text { Environmental } \\
\text { factors } \\
\text { associated } \\
\text { with } \\
\text { adolescent } \\
\text { antisocial } \\
\text { behavior in a } \\
\text { poor urban } \\
\text { community in } \\
\text { Brazil }\end{array}$ & $\begin{array}{l}\text { Identificar os fatores } \\
\text { individuais e ambientais } \\
\text { associados } \\
\text { Comportamento Anti- } \\
\text { Social (ASB) em } \\
\text { adolescentes de uma } \\
\text { comunidade pobre } \\
\text { urbana na periferia da } \\
\text { cidade de São Paulo, } \\
\text { Brasil. }\end{array}$ & $\begin{array}{l}\text { Jovens com altas } \\
\text { pontuações ASB eram mais } \\
\text { propensos a serem vítimas } \\
\text { de punição física grave e ter } \\
\text { um pai ausente ou } \\
\text { substituto. A ansiedade } \\
\text { materna foi um fator de risco } \\
\text { para a depressão apenas } \\
\text { entre os adolescentes mais } \\
\text { velhos. }\end{array}$ \\
\hline 10 & 2012 & $\begin{array}{l}\text { Cad. } \\
\text { Saúde } \\
\text { Pública, } \\
\text { Rio de } \\
\text { Janeiro }\end{array}$ & $\begin{array}{l}\text { Bastos \& } \\
\text { Faerstein }\end{array}$ & $\begin{array}{l}\text { Conceptual } \\
\text { and } \\
\text { methodological } \\
\text { aspects of } \\
\text { relations } \\
\text { between } \\
\text { discrimination } \\
\text { and health in } \\
\text { epidemiologica } \\
\text { I studies }\end{array}$ & $\begin{array}{l}\text { Analisar aspectos } \\
\text { conceituais e } \\
\text { metodológicos das } \\
\text { relações entre } \\
\text { discriminação e saúde } \\
\text { do ponto de vista } \\
\text { epidemiológico. }\end{array}$ & $\begin{array}{l}\text { Como uma fonte } \\
\text { relativamente imprevisível e } \\
\text { incontrolável de estresse } \\
\text { psicossocial, a discriminação } \\
\text { tem sido consistentemente } \\
\text { associada a resultados } \\
\text { adversos para a saúde e } \\
\text { comportamentos, } \\
\text { particularmente transtornos } \\
\text { mentais, tabagismo e uso de } \\
\text { álcool. }\end{array}$ \\
\hline 11 & 2012 & $\begin{array}{l}\text { Internation } \\
\text { al Journal } \\
\text { of Social } \\
\text { Psychiatry }\end{array}$ & Osaka et al. & $\begin{array}{l}\text { Depression } \\
\text { risks in } \\
\text { mothers of } \\
\text { children with } \\
\text { developmental } \\
\text { disabilities: A } \\
\text { cross-cultural } \\
\text { comparison }\end{array}$ & $\begin{array}{l}\text { Explorar o risco de } \\
\text { depressão em mães de } \\
\text { crianças com } \\
\text { deficiências de países } \\
\text { em desenvolvimento } \\
\text { com falta de } \\
\text { profissionais de saúde } \\
\text { mental, realizamos } \\
\text { comparações } \\
\text { interculturais para } \\
\text { quatro países. }\end{array}$ & $\begin{array}{l}\text { Evidencia-se uma relação } \\
\text { entre a situação } \\
\text { socioeconômica e os } \\
\text { números de mães que foram } \\
\text { observados para ter um } \\
\text { elevado grau de sintomas } \\
\text { depressivos. }\end{array}$ \\
\hline 12 & 2012 & $\begin{array}{l}\text { Summa } \\
\text { Psicológic } \\
\text { a UST }\end{array}$ & $\begin{array}{c}\text { Ramos- } \\
\text { Oliveira, } \\
\text { Pankalla \& } \\
\text { Cabecinhas }\end{array}$ & $\begin{array}{l}\text { Ethnic Identity } \\
\text { as predictor for } \\
\text { the well-being: } \\
\text { An exploratory } \\
\text { transcultural } \\
\text { study in Brazil } \\
\text { and Europe }\end{array}$ & $\begin{array}{l}\text { Examinar a associação } \\
\text { entre o bem-estar } \\
\text { subjetivo, malestar com } \\
\text { a etnia em diferentes } \\
\text { grupos étnicos. }\end{array}$ & $\begin{array}{l}\text { Os aspectos sociocognitivos } \\
\text { atuam como eventos } \\
\text { preditores de estresse, por } \\
\text { exemplo, o preconceito e } \\
\text { estereótipos para com } \\
\text { determinados grupos } \\
\text { étnicos, levando a indivíduos } \\
\text { a buscar uma maior } \\
\text { identificação étnica para } \\
\text { minimizar a doença mental e } \\
\text { depressão. }\end{array}$ \\
\hline 13 & 2012 & $\begin{array}{l}\text { Ethnicity \& } \\
\text { Disease }\end{array}$ & $\begin{array}{l}\text { Pavão, } \\
\text { Ploubidis, } \\
\text { Werneck \& } \\
\text { Campos }\end{array}$ & $\begin{array}{l}\text { Racial } \\
\text { discrimination } \\
\text { and health in } \\
\text { Brazil: } \\
\text { Evidence from } \\
\text { a population- } \\
\text { based survey }\end{array}$ & $\begin{array}{l}\text { Investigar a associação } \\
\text { entre a discriminação } \\
\text { racial em negros e } \\
\text { mulatos e três } \\
\text { resultados de saúde: } \\
\text { autopercepção de } \\
\text { saúde (SRH), a } \\
\text { morbidade física e } \\
\text { depressão na } \\
\text { população brasileira. }\end{array}$ & $\begin{array}{l}\text { Uma das consequências da } \\
\text { discriminação racial sobre a } \\
\text { saúde da população } \\
\text { brasileira foi encontrado, } \\
\text { independentemente do } \\
\text { indicador de saúde utilizado, } \\
\text { que revelou que a depressão } \\
\text { foi o resultado de saúde com } \\
\text { a associação mais } \\
\text { pronunciada. }\end{array}$ \\
\hline
\end{tabular}




\begin{tabular}{|c|c|c|c|c|c|}
\hline & Ano & Periódico & Autor & Título & Objetivos \\
\hline 14 & 2012 & $\begin{array}{l}\text { Tempus - } \\
\text { Actas de } \\
\text { Saúde } \\
\text { Coletiva }\end{array}$ & $\begin{array}{c}\text { Silva \& de } \\
\text { Santana }\end{array}$ & $\begin{array}{l}\text { Transtornos } \\
\text { mentais e } \\
\text { pobreza no } \\
\text { Brasil: uma } \\
\text { revisão } \\
\text { sistemática }\end{array}$ & $\begin{array}{l}\text { Avaliar através da } \\
\text { revisão sistemática a } \\
\text { relação entre } \\
\text { transtornos mentais e } \\
\text { situação de pobreza no } \\
\text { Brasil apresentada em } \\
\text { estudos científicos. }\end{array}$ \\
\hline
\end{tabular}

\begin{tabular}{|c|c|c|c|c|c|c|}
\hline 15 & 2013 & $\begin{array}{l}\text { Protestanti } \\
\text { smo em } \\
\text { Revista, } \\
\text { São } \\
\text { Leopoldo }\end{array}$ & $\begin{array}{c}\text { Pereira \& } \\
\text { Pereira }\end{array}$ & $\begin{array}{l}\text { A culpa, o } \\
\text { racismo e a } \\
\text { psicopatologia: } \\
\text { uma reflexão }\end{array}$ & $\begin{array}{l}\text { Analisar teoricamente } \\
\text { sobre a existência de } \\
\text { práticas racistas em } \\
\text { nossa sociedade, a } \\
\text { relação desta } \\
\text { existência com o } \\
\text { sentimento de culpa e o } \\
\text { desencadeamento de } \\
\text { psicopatologias }\end{array}$ & $\begin{array}{l}\text { O racismo aparece como um } \\
\text { dos principais mecanismos } \\
\text { de desvalorização, } \\
\text { humilhação e negligencia de } \\
\text { seres humanos, portanto, } \\
\text { um fator desencadeante de } \\
\text { doenças e sofrimento } \\
\text { psíquico. }\end{array}$ \\
\hline 16 & 2013 & $\begin{array}{c}\text { Health } \\
\text { Expectatio } \\
\text { ns }\end{array}$ & de Freitas & $\begin{array}{l}\text { Aiming for } \\
\text { inclusion: a } \\
\text { case study of } \\
\text { motivations for } \\
\text { involvement in } \\
\text { mental health- } \\
\text { care } \\
\text { governance by } \\
\text { ethnic minority } \\
\text { users }\end{array}$ & $\begin{array}{l}\text { Examinar as } \\
\text { motivações para o } \\
\text { envolvimento na } \\
\text { administração de } \\
\text { cuidados de saúde } \\
\text { mental por usuários de } \\
\text { minorias étnicas } \\
\text { socialmente } \\
\text { desfavorecidos. }\end{array}$ & $\begin{array}{l}\text { O envolvimento dos grupos } \\
\text { minoritários nordestinos na } \\
\text { governança de cuidados de } \\
\text { saúde mental foi conduzido } \\
\text { tanto como motivações } \\
\text { individual como coletivas, } \\
\text { mas a intolerância, vergonha } \\
\text { e, a discriminação causou a } \\
\text { (auto) distanciamento de } \\
\text { muitos usuários de suas } \\
\text { comunidades. }\end{array}$ \\
\hline 17 & 2013 & $\begin{array}{l}\text { Martins et } \\
\text { al. }\end{array}$ & $\begin{array}{c}\text { Martins, dos } \\
\text { Santos \& } \\
\text { Colosso }\end{array}$ & $\begin{array}{l}\text { Relações } \\
\text { étnico-raciais e } \\
\text { psicologia: } \\
\text { publicações } \\
\text { em periódicos } \\
\text { da SciELO e } \\
\text { Lilacs }\end{array}$ & $\begin{array}{l}\text { Revisar o panorama de } \\
\text { como as relações } \\
\text { étnico-raciais tem sido } \\
\text { investigada pela } \\
\text { psicologia. }\end{array}$ & $\begin{array}{l}\text { Indicam que o tema das } \\
\text { relações étnico-raciais tem } \\
\text { sido objeto de investigação } \\
\text { da psicologia, notadamente } \\
\text { da psicologia social, e que a } \\
\text { noção de raça aos poucos } \\
\text { vem sendo incorporada } \\
\text { pelos(as) pesquisadores(as) } \\
\text { desse campo como } \\
\text { categoria de análise para } \\
\text { compreensão das } \\
\text { desigualdades. }\end{array}$ \\
\hline 18 & 2013 & $\begin{array}{l}\text { Cad. } \\
\text { Saúde } \\
\text { Pública, } \\
\text { Rio de } \\
\text { Janeiro }\end{array}$ & $\begin{array}{c}\text { Goto, Couto } \\
\text { \& Bastos }\end{array}$ & $\begin{array}{l}\text { Revisão } \\
\text { sistemática } \\
\text { dos estudos } \\
\text { epidemiológico } \\
\text { s sobre } \\
\text { discriminação } \\
\text { interpessoal e } \\
\text { saúde mental }\end{array}$ & $\begin{array}{l}\text { Analisar estudos } \\
\text { epidemiológicos que } \\
\text { avaliaram a relação } \\
\text { entre discriminação } \\
\text { interpessoal e } \\
\text { condições de saúde } \\
\text { mental }\end{array}$ & $\begin{array}{l}\text { Observaram-se associações } \\
\text { positivas e estatisticamente } \\
\text { significativas entre } \\
\text { discriminação e condições } \\
\text { adversas de saúde mental, } \\
\text { especialmente uso de } \\
\text { substâncias, depressão e } \\
\text { transtornos associados ao } \\
\text { uso de álcool. }\end{array}$ \\
\hline
\end{tabular}




\begin{tabular}{|c|c|c|c|c|c|c|}
\hline & Ano & Periódico & Autor & Título & Objetivos & Principais Resultados \\
\hline 19 & 2013 & $\begin{array}{l}\text { The } \\
\text { Scientific } \\
\text { World } \\
\text { Journal }\end{array}$ & $\begin{array}{l}\text { Bordin, } \\
\text { Pires \& } \\
\text { Paula }\end{array}$ & $\begin{array}{l}\text { Lifetime Paid } \\
\text { Work and } \\
\text { Mental Health } \\
\text { Problems } \\
\text { among poor } \\
\text { Urban 9-to-13- } \\
\text { Year-Old } \\
\text { Children in } \\
\text { Brazil }\end{array}$ & $\begin{array}{l}\text { Verificar se os } \\
\text { problemas emocionais / } \\
\text { comportamentais estão } \\
\text { associados ao trabalho } \\
\text { remunerado em } \\
\text { crianças urbanas } \\
\text { pobres, quando se leva } \\
\text { em conta outros } \\
\text { correlatos potenciais. }\end{array}$ & $\begin{array}{l}\text { Crianças } \\
\text { socioeconomicamente mais } \\
\text { pobres e socialmente } \\
\text { isoladas foram mais } \\
\text { envolvidas no trabalho } \\
\text { remunerado do que seus } \\
\text { pares menos } \\
\text { desfavorecidos. Ademais de } \\
\text { apresentarem níveis de } \\
\text { ansiedade e depressão } \\
\text { quatro vezes mais que } \\
\text { crianças que não estiveram } \\
\text { expostas ao trabalho. }\end{array}$ \\
\hline 20 & 2014 & & $\begin{array}{c}\text { Etcheverry, } \\
\text { Pereira \& } \\
\text { Cordeiro }\end{array}$ & $\begin{array}{l}\text { Depressive } \\
\text { Symptoms and } \\
\text { Suicidal } \\
\text { Ideation in } \\
\text { Adolescents } \\
\text { Accompanying } \\
\text { a Parent in } \\
\text { Recyclable } \\
\text { Trash } \\
\text { Collection }\end{array}$ & $\begin{array}{l}\text { Analisar como estatus } \\
\text { socioeconômico e } \\
\text { vulnerabilidades } \\
\text { psicossociais podem } \\
\text { influenciar em sintomas } \\
\text { depressivos em } \\
\text { adolescentes. }\end{array}$ & $\begin{array}{l}\text { Os adolescentes filhos de } \\
\text { pais catadores de lixo } \\
\text { apresentam sintomas } \\
\text { depressivos e ideação } \\
\text { suicida. }\end{array}$ \\
\hline 21 & 2014 & $\begin{array}{l}\text { Rev. } \\
\text { Panam } \\
\text { Salud } \\
\text { Publica }\end{array}$ & $\begin{array}{c}\text { Barroso, } \\
\text { Melo \& } \\
\text { Guimarães }\end{array}$ & $\begin{array}{l}\text { Depressão em } \\
\text { comunidades } \\
\text { quilombolas no } \\
\text { Brasil: triagem } \\
\text { e fatores } \\
\text { associados }\end{array}$ & $\begin{array}{l}\text { Estimar a prevalência e } \\
\text { fatores associados à } \\
\text { triagem positiva para } \\
\text { episódio depressivo } \\
\text { maior (EDM) em } \\
\text { comunidades } \\
\text { quilombolas do estado } \\
\text { da Bahia, Brasil. }\end{array}$ & $\begin{array}{l}\text { A triagem positiva para EDM } \\
\text { na população quilombola } \\
\text { mostrou-se inferior à triagem } \\
\text { de EDM para outra } \\
\text { população tradicional, a de } \\
\text { índios Karajás, ficando } \\
\text { semelhante à prevalência da } \\
\text { depressão observada para a } \\
\text { população brasileira. Por se } \\
\text { tratar de uma população } \\
\text { historicamente prejudicada e } \\
\text { exposta a várias condições } \\
\text { de vulnerabilidade social, } \\
\text { poderia se esperar uma } \\
\text { maior triagem positiva para } \\
\text { depressão. }\end{array}$ \\
\hline 22 & 2014 & $\begin{array}{l}\text { Cad. } \\
\text { Saúde } \\
\text { Pública, } \\
\text { Rio de } \\
\text { Janeiro }\end{array}$ & $\begin{array}{l}\text { Gonçalves } \\
\text { et al. }\end{array}$ & $\begin{array}{l}\text { Brazilian } \\
\text { multicentre } \\
\text { study of } \\
\text { common } \\
\text { mental } \\
\text { disorders in } \\
\text { primary care: } \\
\text { rates and } \\
\text { related }\end{array}$ & $\begin{array}{l}\text { Avaliar a taxa de } \\
\text { transtornos mentais } \\
\text { comuns e suas } \\
\text { associações com } \\
\text { características } \\
\text { sociodemográficas em } \\
\text { unidades de saúde da } \\
\text { família }\end{array}$ & $\begin{array}{l}\text { Problemas de saúde mental } \\
\text { foram especialmente altos } \\
\text { em mulheres, } \\
\text { desempregados, em } \\
\text { pessoas com baixa } \\
\text { escolaridade e com baixa } \\
\text { renda. }\end{array}$ \\
\hline 23 & 2014 & $\begin{array}{c}\text { Revista de } \\
\text { Psicologia } \\
\text { da } \\
\text { UNESP }\end{array}$ & $\begin{array}{l}\text { Lages, } \\
\text { Tavares, } \\
\text { Santos, } \\
\text { Carvalho \& } \\
\text { Maciel }\end{array}$ & $\begin{array}{l}\text { Pesquisas em } \\
\text { Psicologia } \\
\text { Social no } \\
\text { Campo da } \\
\text { Saúde da } \\
\text { População } \\
\text { Negra no } \\
\text { Brasil }\end{array}$ & $\begin{array}{l}\text { Revisar no campo da } \\
\text { Psicologia Social e } \\
\text { saúde da população } \\
\text { negra, no Brasil, } \\
\text { considerando que os } \\
\text { coletivos negros sofrem } \\
\text { desigualdades sócio- } \\
\text { historicamente } \\
\text { determinadas e, dentre } \\
\text { elas, doenças e } \\
\text { agravos que são }\end{array}$ & $\begin{array}{l}\text { Apontou para uma baixa } \\
\text { produção da Psicologia } \\
\text { Social no referido tema, o } \\
\text { que limita sua atuação na } \\
\text { luta pelos direitos sociais e } \\
\text { sua participação nas } \\
\text { políticas públicas sobre } \\
\text { saúde. }\end{array}$ \\
\hline
\end{tabular}




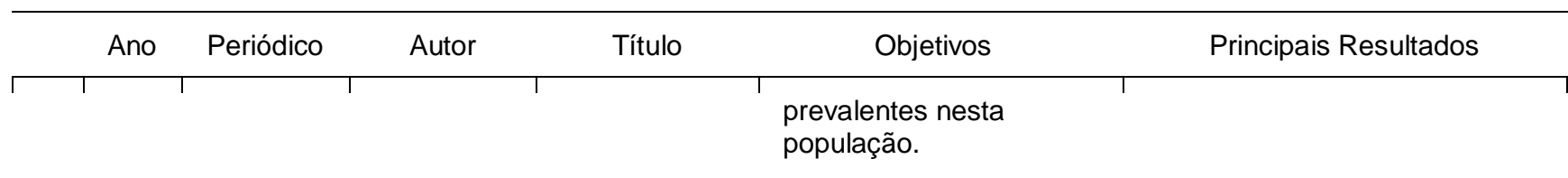

\begin{tabular}{|c|c|c|c|c|c|c|}
\hline 24 & 2014 & $\begin{array}{l}\text { Cad. } \\
\text { Saúde } \\
\text { Pública, } \\
\text { Rio de } \\
\text { Janeiro }\end{array}$ & $\begin{array}{c}\text { Bastos, } \\
\text { Barros, } \\
\text { Celeste, } \\
\text { Paradies \& } \\
\text { Faerstein }\end{array}$ & $\begin{array}{l}\text { Age, class and } \\
\text { race } \\
\text { discrimination: } \\
\text { their } \\
\text { interactions } \\
\text { and } \\
\text { associations } \\
\text { with mental } \\
\text { health among } \\
\text { Brazilian } \\
\text { university } \\
\text { students }\end{array}$ & $\begin{array}{l}\text { Explorar diferentes } \\
\text { tipos de discriminação, } \\
\text { suas interações e } \\
\text { associações com } \\
\text { transtornos mentais } \\
\text { comuns em } \\
\text { universitários do Rio de } \\
\text { Janeiro, Brasil. }\end{array}$ & $\begin{array}{l}\text { Discriminação por idade, } \\
\text { classe e cor/raça foram as } \\
\text { mais frequentemente } \\
\text { relatadas. No modelo } \\
\text { totalmente ajustado, } \\
\text { discriminação atribuída à } \\
\text { cor/raça e classe foram } \\
\text { ambas associadas com odds } \\
\text { aumentadas de transtornos } \\
\text { mentais comuns }\end{array}$ \\
\hline 25 & 2014 & $\begin{array}{l}\text { Saúde } \\
\text { Soc. São } \\
\text { Paulo }\end{array}$ & $\begin{array}{l}\text { Barros } \\
\text { Baptista, } \\
\text { Dellosi \& } \\
\text { Escuder }\end{array}$ & $\begin{array}{l}\text { Censo } \\
\text { psicossocial } \\
\text { dos moradores } \\
\text { em hospitais } \\
\text { psiquiátricos } \\
\text { do estado de } \\
\text { São Paulo: um } \\
\text { olhar sob a } \\
\text { perspectiva } \\
\text { racial }\end{array}$ & $\begin{array}{l}\text { Verificar o perfil dos } \\
\text { moradores dos } \\
\text { hospitais psiquiátricos } \\
\text { do Estado de São } \\
\text { Paulo segundo raça/co }\end{array}$ & $\begin{array}{l}\text { Constatou-se uma maior } \\
\text { proporção de negros que } \\
\text { estão internados porque não } \\
\text { têm renda e/ou lugar para } \\
\text { morar. Os efeitos } \\
\text { psicossociais do racismo e o } \\
\text { impacto dos processos de } \\
\text { preconceito, exclusão e } \\
\text { apartamento social na saúde } \\
\text { mental são evidenciados. }\end{array}$ \\
\hline 26 & 2015 & $\begin{array}{c}\mathrm{J} \\
\text { Communit } \\
\text { y Health }\end{array}$ & $\begin{array}{l}\text { Franco, } \\
\text { Ferreira, } \\
\text { Vieira \& } \\
\text { Silva }\end{array}$ & $\begin{array}{l}\text { Ethnicity, } \\
\text { Obesity and } \\
\text { Emotional } \\
\text { Factors } \\
\text { Associated } \\
\text { With } \\
\text { Gestational } \\
\text { Hypertension }\end{array}$ & $\begin{array}{l}\text { Identificar os fatores } \\
\text { que podem estar } \\
\text { relacionados com a } \\
\text { ocorrência de } \\
\text { hipertensão arterial } \\
\text { gestacional. }\end{array}$ & $\begin{array}{l}\text { Os fatores como a raça não- } \\
\text { branca, depressão, náuseas, } \\
\text { obesidade e ansiedade } \\
\text { podem ser consideradas } \\
\text { fatores de risco para a } \\
\text { ocorrência de hipertensão } \\
\text { arterial gestacional. }\end{array}$ \\
\hline 27 & 2015 & $\begin{array}{l}\text { Epidemiol. } \\
\text { Serv. } \\
\text { Saúde }\end{array}$ & $\begin{array}{l}\text { Massignam, } \\
\text { Bastos \& } \\
\text { Nedel }\end{array}$ & $\begin{array}{l}\text { Discriminação } \\
\text { e saúde: um } \\
\text { problema de } \\
\text { acesso }\end{array}$ & $\begin{array}{l}\text { Estimular estudos e } \\
\text { ações sobre os } \\
\text { processos de } \\
\text { discriminação no } \\
\text { âmbito da atenção à } \\
\text { saúde. }\end{array}$ & $\begin{array}{l}\text { Mostram-se necessárias } \\
\text { mais pesquisas sobre a } \\
\text { ocorrência de discriminação } \\
\text { e seus efeitos sobre a oferta, } \\
\text { o acesso e a qualidade na } \\
\text { atenção à saúde de } \\
\text { segmentos da população } \\
\text { mais vulneráveis ao estigma } \\
\text { ou preconceito, } \\
\text { marginalizados pela } \\
\text { sociedade. }\end{array}$ \\
\hline 28 & 2015 & $\begin{array}{l}\text { Rev. Bras } \\
\text { Epidemiol. }\end{array}$ & $\begin{array}{c}\text { Barroso, } \\
\text { Melo \& } \\
\text { Guimarães }\end{array}$ & $\begin{array}{l}\text { Fatores } \\
\text { associados à } \\
\text { depressão: } \\
\text { diferenças por } \\
\text { sexo em } \\
\text { moradores de } \\
\text { comunidades } \\
\text { Quilombolas }\end{array}$ & $\begin{array}{l}\text { Investigar os fatores } \\
\text { associados à } \\
\text { depressão para } \\
\text { homens e mulheres }\end{array}$ & $\begin{array}{l}\text { Para os homens, os fatores } \\
\text { associados à depressão } \\
\text { foram diagnóstico prévio de } \\
\text { doença crônica, } \\
\text { autoavaliação de saúde } \\
\text { ruim/muito ruim e acesso } \\
\text { ruim aos serviços de saúde. } \\
\text { Para as mulheres, os fatores } \\
\text { associados foram } \\
\text { diagnóstico prévio de } \\
\text { transtorno psiquiátrico, } \\
\text { autoavaliação de saúde } \\
\text { ruim/muito ruim, histórico de }\end{array}$ \\
\hline
\end{tabular}




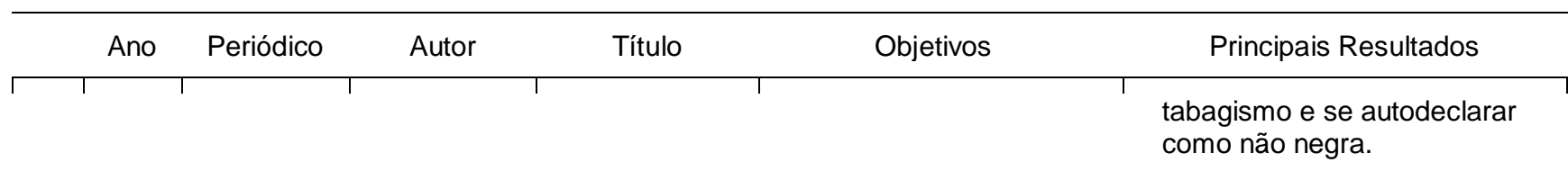

\begin{tabular}{|c|c|c|c|c|c|c|}
\hline 29 & 2016 & $\begin{array}{l}\text { Rev. Esc. } \\
\text { Enferm. } \\
\text { USP }\end{array}$ & Paiva et al. & $\begin{array}{l}\text { Sofrimento } \\
\text { psíquico e a } \\
\text { abordagem da } \\
\text { comunidade } \\
\text { na voz do } \\
\text { agente } \\
\text { comunitário de } \\
\text { saúde }\end{array}$ & $\begin{array}{l}\text { Descrever como as } \\
\text { pessoas em sofrimento } \\
\text { psíquico são percebidas } \\
\text { pela comunidade na voz } \\
\text { do agente comunitário } \\
\text { de saúde. }\end{array}$ & $\begin{array}{l}\text { A rejeição da pessoa que } \\
\text { adoeceu é observada como } \\
\text { uma reação bastante } \\
\text { comum, acompanhada do } \\
\text { preconceito e discriminação, } \\
\text { marginalizando-a da } \\
\text { sociedade. O indivíduo não é } \\
\text { mais reconhecido pelo } \\
\text { nome, em algumas } \\
\text { circunstâncias, perde sua } \\
\text { identidade. }\end{array}$ \\
\hline 30 & 2016 & $\begin{array}{c}\text { J. of } \\
\text { affective } \\
\text { Disorders }\end{array}$ & $\begin{array}{c}\text { Filha, } \\
\text { Ayers, da } \\
\text { Gama \& do } \\
\text { Carmo Leal }\end{array}$ & $\begin{array}{l}\text { Factors } \\
\text { associated } \\
\text { with } \\
\text { postpartum } \\
\text { depressive } \\
\text { symptomatolo } \\
\text { gy in Brazil: } \\
\text { The Birth in } \\
\text { Brazil National } \\
\text { Research } \\
\text { Study, } \\
2011 / 2012\end{array}$ & $\begin{array}{l}\text { Examinar o impacto } \\
\text { sociodemográficos } \\
\text { individual e fatores de } \\
\text { risco obstétricos em } \\
\text { depressão pós-parto. }\end{array}$ & $\begin{array}{l}\text { A prevalência da depressão } \\
\text { pós-parto é elevada em } \\
\text { mulheres brasileiras seis } \\
\text { meses após o nascimento. A } \\
\text { qualidade do atendimento é } \\
\text { pobre nas mulheres e nos } \\
\text { bebês. }\end{array}$ \\
\hline 31 & 2016 & $\begin{array}{l}\text { J. Hum. } \\
\text { Growth } \\
\text { Dev. }\end{array}$ & $\begin{array}{l}\text { de Lima } \\
\text { Garcia, } \\
\text { Albuquer- } \\
\text { que, Drezett } \\
\text { \& Adami }\end{array}$ & $\begin{array}{l}\text { Health of } \\
\text { sexual } \\
\text { minorities in } \\
\text { north-eastern } \\
\text { Brazil: } \\
\text { representation } \\
\text { s, behaviours } \\
\text { and obstacles }\end{array}$ & $\begin{array}{l}\text { Identificar as } \\
\text { Representações sociais } \\
\text { (RS) de Saúde e de } \\
\text { grandes desigualdades, } \\
\text { obstáculos e desafios } \\
\text { vividos por lésbicas, } \\
\text { gays, bissexuais, } \\
\text { transgêneros e sobre o } \\
\text { acesso aos serviços de } \\
\text { saúde. }\end{array}$ & $\begin{array}{l}\text { A maioria dos participantes } \\
\text { acreditava na ausência de } \\
\text { doença e indicou a } \\
\text { vulnerabilidade do grupo } \\
\text { para as doenças } \\
\text { sexualmente transmissíveis. } \\
\text { As dificuldades estavam } \\
\text { relacionadas com as } \\
\text { deficiências do serviço de } \\
\text { saúde e do preconceito. }\end{array}$ \\
\hline 32 & 2016 & $\begin{array}{l}\text { Ciência \& } \\
\text { Saúde } \\
\text { Coletiva }\end{array}$ & $\begin{array}{l}\text { Portugal, } \\
\text { Campos, } \\
\text { Gonçalves, } \\
\text { de Jesus } \\
\text { Mari \& } \\
\text { Fortes }\end{array}$ & $\begin{array}{l}\text { Qualidade de } \\
\text { vida em } \\
\text { pacientes da } \\
\text { atenção } \\
\text { primária do } \\
\text { Rio de Janeiro } \\
\text { e São Paulo, } \\
\text { Brasil: } \\
\text { associações } \\
\text { com eventos } \\
\text { de vida } \\
\text { produtores de } \\
\text { estresse e } \\
\text { saúde mental }\end{array}$ & $\begin{array}{l}\text { Identificar a associação } \\
\text { entre variáveis } \\
\text { demográficas, } \\
\text { socioeconômicas, } \\
\text { transtorno mental } \\
\text { comum, sintomas } \\
\text { sugestivos de } \\
\text { ansiedade e depressão }\end{array}$ & $\begin{array}{l}\text { Foram encontradas } \\
\text { associações do domínio } \\
\text { físico com os problemas de } \\
\text { saúde e discriminação, do } \\
\text { psicológico com } \\
\text { discriminação, das relações } \\
\text { sociais com problemas } \\
\text { financeiros/estruturais, } \\
\text { causas externas e } \\
\text { problemas de saúde, e do } \\
\text { meio ambiente com } \\
\text { problemas financeiros/ } \\
\text { estruturais, causas externas } \\
\text { e discriminação }\end{array}$ \\
\hline
\end{tabular}

\section{Conclusão}

Os processos relativos as cognições sociais apresentam-se como um evento traumático e estressante para alguns grupos étnicos e minoritários, provocando uma série de sofrimento psíquico nos indivíduos.
Os estudos sugerem que muitos são os fatores que podem levar a um grupo étnico ou minoritário a desenvolver um estado de ansiedade, depressão e/ou outros transtornos mentais em função dos eventos estressantes vivenciados como as 
adversidades do ambiente e a vida social (ex. desemprego, separação dos pais em idades iniciais, habitação inadequada, experiência de racismo) são considerados como fatores de risco para 0 desenvolvimento de transtornos mentais, tanto para os nativos de um país como para imigrantes (Ramos-Oliveira, Techio, Paéz \& Herranz, 2005; Wessely, Castle, Der \& Murray, 1991).

Viswanath (2006) aponta que há uma prevalência em subestimar o impacto das desigualdades sociais integradas ao conhecimento e à comunicação no agravamento das desigualdades em saúde. Uma carga importante de morbidades está associada a comportamentos potencialmente modificáveis por meio de oportunidades adequadas e de acesso a cuidados preventivos e a informação em saúde (Williams \& Priest, 2015).

Observou-se que algumas práticas e condutas profissionais erguem uma série de procedimentos que comprometem a probidade, e consequentemente a invisibilidade de determinadas doenças que afetam de forma mais significativa a um determinado seguimento da população, adicionalmente, a não inclusão da questão étnico/racial na formação médica e de equipes de saúde denota as consequências reveladas nos relatos dos participantes de alguns estudos analisados.

Por outro lado, a escassez de pesquisas no âmbito da psicologia evidencia uma lacuna que necessita ser preenchida, especialmente a Psicologia social. Lages et al. (2014) argumentam que as doenças físicas afetam o corpo do sujeito, porém os preconceitos, o desmerecimento das identidades culturais, afetam as subjetividades, e esse afetamento pode alcançar o sujeito psíquico provocando depressão, desmotivação e baixa autoestima.

De acordo com Sawaia (2008), a experiência emocional de quem se vê impedido de expressar-se em todo o seu potencial humano, por estar condicionado a qualquer tipo de modalidade processual de exclusão e de sofrimento éticopolítico. Segundo a autora, esse sofrimento é a dor mediada pelas injustiças sociais.

Faz -se necessário observar que determinados problemas metodológicos prejudicam a fidedignidade dos dados (Simpson et al., 2004), como o estudo de Barroso, Melo y Guimarães (2014) cuja a triagem positiva para EDM na população quilombola (12\%) mostrou-se semelhante à prevalência da depressão observada para a população brasileira, mesmo tratando-se de uma população historicamente prejudicada e exposta a várias condições de vulnerabilidade social. $O$ esperado seria uma maior triagem positiva para depressão, mas segundo os autores devem-se considerar as características das medidas de autorelato em populações vulneráveis que podem levar à uma subidentificação de patologias que dependem da habilidade dos indivíduos para reconhecer sinais e sintomas em si ou recordar um diagnóstico prévio.

Foi possível observar com este artigo a relação do ponto de vista empírico entre a etnia/raça assim como o status com a ocorrência de transtornos psicológicos, todavia necessitam de mais pesquisas de maneira a criar uma consistência, e consequentemente desenvolver intervenções e políticas públicas que abarquem estas questões. Igualmente, faz-se necessário mais estudo empírico na área da psicologia de maneira que evidencie 0 comprometimento psíquico dos indivíduos relacionados com sua pertença étnica e a iniquidade no tratamento de saúde que fomenta um desconforto mental.

\section{Referências}

Asnaani, A., Richey, J., Dimaite, M., Hinton, D. \& Hofmann, S. (2010). A cross-ethnic comparison of lifetime prevalence rates of anxiety disorders. The Journal of Nervous and Mental Disease, 198(8), 551-555. doi:10.1097/NMD.0b013e3181ea169f

Barros, S., Batista, L., Dellosi, M. \& Escuder, M. (2014). Censo psicossocial dos moradores em hospitais psiquiátricos do estado de São Paulo: um olhar sob a perspectiva racial. Saúde e Sociedade, 23(4), 1235-1247. doi:10.1590/S010412902014000400010

Barroso, S., Melo, A. \& Guimarães, M. (2014). Depressão em comunidades quilombolas no Brasil: triagem e fatores associados. Revista Panamericana de Salud Pública, 35(4), 256-263. Recuperado de http://www.scielosp.org/pdf/rpsp/v35n4/04.pdf

Barroso, S., Melo, A. \& Guimarães, M. (2015). Factors associated with depression: sex differences between residents of Quilombo communities. Revista Brasileira de Epidemiologia, 18(2), 503514. doi:10.1590/1980-5497201500020017

Bastos, J. \& Faerstein, E. (2012). Conceptual and methodological aspects of relations between discrimination and health in epidemiological studies. Cadernos de saúde pública, 28(1), 177183. doi:10.1590/S0102-311X2012000100019

Bastos, J., Barros, A., Celeste, R., Paradies, Y. \& Faerstein, E. (2014). Age, class and race discrimination: their interactions and associations with mental health among Brazilian university students. Cadernos de Saúde Pública, 30(1), 175-186. doi:10.1590/0102-311X00163812

Bastos, J., Celeste, R., Faerstein, E. \& Barros, A. (2010). Discriminação racial e saúde: uma revisão sistemática de escalas com foco em suas propriedades. Saúde \& Transformação Social/Health \& Social Change, 70(7), 10911099. doi:10.1016/j.socscimed.2009.12.020

Berlim, M., McGirr, A. \& Fleck, M. (2008). Can sociodemographic and clinical variables predict the quality of life of outpatients with major depression?. Psychiatry research, 160(3), 364371. doi:10.1016/j.psychres.2007.07.024

Blazer, D., Kessler, R., McGonagle, K. \& Swartz, M. (1994). The prevalence and distribution of major depression in a national community sample: The National Comorbidity Survey. American Journal 
of Psychiatry, 151(7), 979-986. doi:10.1176/ajp.151.7.979

Bordin, I., Pires, I. \& Paula, C. (2013). Lifetime paid work and mental health problems among poor urban 9-to-13-year-old children in Brazil. The Scientific World Journal, 1-7. doi:10.1155/2013/815218

Brown, D., Eaton, W. \& Sussman, L. (1990). Racial differences in prevalence of phobic disorders. Journal of Nervous and Mental Disease, 178(7), 434-441. Recuperado de https://www.ncbi.nlm.nih.gov/pubmed/2366057

Curto, B., Paula, C., do Nascimento, R., Murray, J. \& Bordin, I. (2011). Environmental factors associated with adolescent antisocial behavior in a poor urban community in Brazil. Social psychiatry and Psychiatric epidemiology, 46(12), 1221-1231. doi:10.1007/s00127-010-0291-2

Etcheverry, G., Pereira, É. \& Cordeiro, M. (2014). Depressive symptoms and suicidal ideation in adolescents accompanying a parent in recyclable trash collection. The Spanish Journal of Psychology, 17(13),1-8. doi:10.1017/sjp.2014.13

Faro, A. \& Pereira, M. (2011). Raça, racismo e saúde: a desigualdade social da distribuição do estresse. Estudos de Psicologia, 16(3), 271-278. doi:10.1590/S1413-294X2011000300009

Ferriolli, S., Marturano, E. \& Puntel, L. (2007). Contexto familiar e problemas de saúde mental infantil no Programa Saúde da Família. Revista de Saúde Pública, 41(2), 251-259. doi:10.1590/S003489102006005000017

Filha, M., Ayers, S., da Gama, S. \& do Carmo Leal, M. (2016). Factors associated with postpartum depressive symptomatology in Brazil: The Birth in Brazil National Research Study, 2011/2012. Journal of affective disorders, 194, 159-167. doi:10.1016/j.jad.2016.01.020

Fonseca-Machado, M., Alves, L., dos Santos Monteiro, J., Stefanello, J., Nakano, A., Haas, V. \& GomesSponholz, F. (2015). Depressive disorder in pregnant Latin women: does intimate partner violence matter?. Journal of Clinical Nursing, 24(9-10), 1289-1299. doi:10.1111/jocn.12728

Franco, R., Ferreira, C., Vieira, C. \& Silva, R. (2015). Ethnicity, obesity and emotional factors associated with gestational hypertension. Journal of Community Health, 40(5), 899-904. doi:10.1007/s10900-015-0010-8

Freitas de, C. (2015). Aiming for inclusion: a case study of motivations for involvement in mental health-care governance by ethnic minority users. Health Expectations, 18(5), 1093-1104. doi:10.1111/hex.12082

Gonçalves, D., Mari, J., Bower, P., Gask, L., Dowrick, C., Tófoli, L. \& Fortes, S. (2014). Brazilian multicentre study of common mental disorders in primary care: rates and related social and demographic factors. Cadernos de Saúde Pública, 30(3), 623-632. doi:10.1590/0102$311 \times 00158412$

Goto, J., Couto, P. \& Bastos, J. (2013). Revisão sistemática dos estudos epidemiológicos sobre discriminação interpessoal e saúde mental. Cad. Saúde Pública, 29(3), 445-459. Recuperado de http://www.scielo.br/pdf/csp/v29n3/a04v29n3.pd

Juang, L., Ittel, A., Hoferichter, F. \& Gallarin, M. (2016). Perceived racial/ethnic discrimination and adjustment among ethnically diverse college students: family and peer support as protective factors. Journal of College Student Development, 57(4), 380-394. doi:10.1353/csd.2016.0048

Kang, S., Razzouk, D., Mari, J. \& Shirakawa, I. (2009). The mental health of Korean immigrants in São Paulo, Brazil. Cadernos de Saúde Pública, 25(4), 819-826. Recuperado http://www.scielo.br/pdf/csp/v25n4/13.pdf

Kelaher, M., Paul, S., Lambert, H., Ahmad, W. \& Smith, G. (2008). Discrimination and health in an English study. Social Science \& Medicine, 66(7), 16271636. doi:10.1016/j.socscimed.2007.12.005

Kessler, R., McGonagle, K., Zhao, S., Nelson, C., Hughes, M. \& Eshleman, S. (1994). Lifetime and 12-month prevalence of DSM-III-R psychiatric disorders in the National Comorbidity Survey. Archives of General Psychiatry, 51(1), 8-19. doi:10.1001/archpsyc.1994.03950010008002

Krieger, N. (1999). Embodying inequality: A review of concepts, measures, and methods for studying health consequences of discrimination. International Journal of Health Services, 29(2), 295-352. doi:10.2190/M11W-VWXE-KQM9G97Q

Krieger, N. (2003). Does racism harm health? Did child abuse exist before 1962? On explicit questions, critical science, and current controversies: an ecosocial perspective. American Journal of Public Health, 93(2), 194-199. doi:10.2105/AJPH.93.2.194

Lages, S., Tavares, N., Santos, S., Carvalho, M. \& Maciel, L. (2014). Pesquisas em Psicologia Social no Campo da Saúde da População Negra no Brasil. Revista de Psicologia da UNESP, 13(1), 1-10. Recuperado de http://pepsic.bvsalud.org/pdf/revpsico/v13n1/a01 .pdf

Lima Garcia de, C., Albuquerque, G., Drezett, J. \& Adami, F. (2016). Health of Sexual Minorities in Northeastern Brazil: Representations, Behaviours and Obstacles. Journal of Human Growth and Development, 26(1), 94-100. doi:10.7322/jhgd.110985

Martins, E., dos Santos, A. \& Colosso, M. (2013). Relações étnico-raciais e psicologia: publicações em periódicos da SciELO e Lilacs. Psicologia: teoria e prática, 15(3), 118-133. Recuperado de http://editorarevistas.mackenzie.br/index.php/pt p/article/view/4919/4598

Massignam, F., Bastos, J. \& Nedel, F. (2015). Discriminação e saúde: um problema de acesso. Epidemiologia e Serviços de Saúde, 24(3), 541544. doi:10.5123/S1679-49742015000300020

McLaughlin, K., Hilt, L. \& Nolen-Hoeksema, S. (2007). Racial/ethnic differences in internalizing and externalizing symptoms in adolescents. Journal 
of Abnormal Child Psychology, 35(5), 801-816. doi:10.1007/s10802-007-9128-1

Miyasaka, L., Canasiro, S., Abe, Y., Otsuka, K., Tsuji, K., Hayashi, T. \& Kato, S. (2007). Migration and mental health: Japanese Brazilians in Japan and in Brazil. Jornal Brasileiro de Psiquiatria, 56(1), 48-52. doi:10.1590/S0047-20852007000100011

Nakamura, B., Ebesutani, C., Bernstein, A. \& Chorpita, B. (2009). A psychometric analysis of the Child Behavior Checklist DSM Oriented scales. Journal of Psychopathology and Behavioral Assessment $31(3)$, 178-189. doi:10.1007/s10862-008-9119-8

Paiva, P., de Torrenté, M., Landim, F., de Oliveira Branco, J., Tamboril, B. \& Cabral, A. (2016). Sofrimento psíquico e a abordagem da comunidade na voz do agente comunitário de saúde. Revista da Escola de Enfermagem da USP, 50(spe), 139144. doi:10.1590/S0080-623420160000300020

Paradies, Y. \& Williams, D. (2008). Racism and Health. En K. Heggenhougen \& S. Quah (Eds.), International Encyclopedia Public Health (pp. 474-482). San Diego: Academic Press.

Pascoe, E. \& Smart, L. (2009). Perceived discrimination and health: a meta-analytic review. Psychological Bulletin, 135(4), 531-554. doi:10.1037/a0016059

Paula, C., Bordin, I., Mari, J., Velasque, L., Rohde, L. \& Coutinho, E. (2014). The mental health care gap among children and adolescents: data from an epidemiological survey from four Brazilian regions. Plos One, 9(2). doi:10.1371/journal.pone. 0088241

Pavão, A., Ploubidis, G., Werneck, G. \& Campos, M. (2012). Racial discrimination and health in Brazil: evidence from a population-based survey. Ethnicity \& disease, 22(3), 353-359. Recuperado de

https://www.ethndis.org/edonline/index.php/ethn dis/article/view/462/511

Portugal, F., Campos, M., Gonçalves, D., de Jesus Mari, J. \& Fortes, S. (2016). Qualidade de vida em pacientes da atenção primária do Rio de Janeiro e São Paulo, Brasil: associações com eventos de vida produtores de estresse e saúde mental. Ciência \& Saúde Coletiva, 21(2), 497-508. doi:10.1590/1413-81232015212.20032015

Ramos-Oliveira, D. (2009). Identidad étnica, Autoestima colectiva, Valores y Bienestar: Estudios en Brasil, Argentina, Polonia y Portugal (Tesis doctoral, Universidad del País Vasco, España).

Ramos-Oliveira, D., Pankalla, A. \& Cabecinhas, R. (2012). Ethnic Identity as predictor for the well-being: An exploratory transcultural study in Brazil and Europe. Summa Psicológica UST, 9(2), 33-42. Recuperado http://www.summapsicologica.cl/index.php/sum ma/article/view/96/98

Ramos-Oliveira, D., Techio, E., Paéz, D. \& Herráz, K. (2005). Factores Predictores de las actitudes ante la Inmigración. Revista Psicología Social, 20(1), 19-37. doi:10.1174/0213474052871060
Riolo, S., Nguyen, T., Greden, J. \& King, C. (2005). Prevalence of depression by race/ethnicity: findings from the National Health and Nutrition Examination Survey III. American Journal of Public Health, 95(6), 998-1000. doi:10.2105/AJPH.2004.047225

Sawaia, B. (2008). O sofrimento ético-político como categoria de análise da dialética exclusão/inclusão. In B. Sawaia (Org.), As artimanhas da exclusão: análise psicossocial e ética da desigualdade social (pp. 97-118). Coleção Psicologia Social: Editora Vozes Ltda.

Silva, D. \& de Santana, P. (2012). Transtornos mentais e pobreza no Brasil: uma revisão sistemática. Tempus Actas de Saúde Coletiva, 6(4), 175-185. doi:10.18569/tempus.v6i4.1214

Vilhena, J. (2006). A violência da cor: sobre racismo, alteridade e intolerância. Revista Psicologia Política, 6(12), 2. Recuperado de http://www.fafich.ufmg.br/ psicopol/seer/ojs/vie warticle. php?id=7\&layout $=$ html

Viswanath, K. (2006). Public communications and its role in reducing and elimination health disparities. In G. Thomson, F. Mitchell \& M. Williams (Eds.), Examining the health disparities research plan of the National Institutes of Health: Unfinished Business (pp. 215-253). Washington: National Academies Press.

Watkins, D., Assari, S. \& Johnson-Lawrence, V. (2015). Race and ethnic group differences in comorbid major depressive disorder, generalized anxiety disorder, and chronic medical conditions. Journal of racial and ethnic health disparities, 2(3), 385394. doi:10.1007/s40615-015-0085-z

Watson, K., Roberts, N. \& Saunders, M. (2012). Factors Associated with Anxiety and Depression among African American and White Women. International Scholarly Research Network, 1-8. doi:10.5402/2012/43232

Wessely, S., Castle D., Der, G. \& Murray, R. (1991). Schizophrenia and Afro-Caribbeans. A casecontrol study. The British Journal of Psychiatry, 159(6),795-801. doi:10.1192/bjp.159.6.795

Williams, D. \& Priest, N. (2015). Racismo e Saúde: um corpus crescente de evidência internacional. Sociologias, 17(40), doi:10.1590/15174522-017004004

124-174.

Williams, D. \& Mohammed, S. (2009). Discrimination and racial disparities in health: evidence and needed research. Journal of Behavioral Medicine, 32(1), 20-47. doi:10.1007/s10865-008-9185-0 